\title{
Direct Josephson coupling between superconducting flux qubits
}

M. Grajcar, ${ }^{1,2}$ A. Izmalkov, ${ }^{1,3}$ S. H. W. van der Ploeg,,${ }^{1,4}$ S. Linzen, ${ }^{1}$ E. Il'ichev,,${ }^{1} *$ Th. Wagner, ${ }^{1}$ U. Hübner, ${ }^{1}$ H.-G. Meyer, ${ }^{1}$ Alec Maassen van den Brink, ${ }^{5, \dagger}$ S. Uchaikin,,${ }^{1,5}$ and A. M. Zagoskin ${ }^{5,6, \$}$

${ }^{1}$ Institute for Physical High Technology, P.O. Box 100239, D-07702 Jena, Germany

${ }^{2}$ Department of Solid State Physics, Comenius University, SK-84248 Bratislava, Slovakia

${ }^{3}$ Moscow Engineering Physics Institute (State University), Kashirskoe shosse 31, 115409 Moscow, Russia

${ }^{4} M E S A+$ Research Institute and Faculty of Science and Technology, University of Twente, P.O. Box 217, 7500 AE Enschede, The Netherlands

${ }^{5}$ D-Wave Systems Inc., 320-1985 W. Broadway, Vancouver, British Columbia, Canada V6J 4 Y3

${ }^{6}$ Physics and Astronomy Department, The University of British Columbia, 6224 Agricultural Road, Vancouver, British Columbia, Canada V6T 1 Z1

(Received 2 April 2005; published 14 July 2005)

\begin{abstract}
We have demonstrated strong antiferromagnetic coupling between two three-junction flux qubits based on a shared Josephson junction, and therefore not limited by the small inductances of the qubit loops. The coupling sign and magnitude were measured by coupling the system to a high-quality superconducting tank circuit. Design modifications allowing to continuously tune the coupling strength and/or make the coupling ferromagnetic are discussed.
\end{abstract}

\section{DOI: 10.1103/PhysRevB.72.020503}

Quantum superposition of macroscopic states was conclusively demonstrated in superconducting Josephson structures in 2000 (Ref. 1). Such structures are natural candidates for the role of qubits (quantum bits), the constituent elements of quantum computers. Successful operation of a quantum computer would be the ultimate confirmation of the validity of quantum mechanics on the macroscopic scale, which makes the task of controllably linking a significant number of qubits more than just an advance in technology.

The coupling energy $J$ must be comparable to the splittings between the two lowest eigenstates of individual qubits. On the other hand, the coupling must not excite the qubits to higher levels, or significantly increase the qubits' interaction with undesirable degrees of freedom, leading to decoherence and dissipation. Finally, $J$ should be either variable by design or, even better, tunable during the system's operation.

We demonstrate the coupling of two three-Josephsonjunction (3JJ) flux qubits, making progress towards meeting these requirements, and discuss the ways of its further improvement. The 3JJ qubit consists of a superconducting loop with small inductance $L$ interrupted by three Josephson junctions. The two different directions of persistent current in the loop form the qubit's basis states. ${ }^{2}$ The 3JJ design enables classical bistability even for $L \rightarrow 0$, resulting in a weak coupling to environmental magnetic-flux noises. As a result, quantum behavior with long decoherence times was observed in this type of qubit by several groups. ${ }^{3-5}$

However, their small $L$ makes it difficult to couple $3 \mathrm{JJ}$ qubits inductively; generally, $J$ is smaller than the singlequbit level splitting. We therefore implement the proposals 6,7 to directly link two qubits through a shared junction (Fig. 1). The resulting coupling not only is strong, but can also be varied independently of other design parameters by choosing the shared junction's size.

To calculate $J$, we neglect the inductances so that the potential term in the Hamiltonian contains only the Josephson energy $U_{\mathrm{J}}=-\sum_{j=0}^{6} E_{j} \cos \phi_{j}$, and use flux quantization, $\phi_{1}+\phi_{2}+\phi_{3}+\phi_{0}=2 \pi\left(\frac{1}{2}+f_{a}\right)$ and $\phi_{4}+\phi_{5}+\phi_{6}-\phi_{0}=2 \pi\left(\frac{1}{2}+f_{b}\right)$,
PACS number(s): 74.50. $+\mathrm{r}$, 85.25.Am, 85.25.Cp

to eliminate $\phi_{2 ; 5}\left(f_{a ; b}=\Phi_{a ; b}^{\mathrm{x}} / \Phi_{0}-\frac{1}{2}\right.$ is the reduced flux bias). In the simplest case $\left[f_{a ; b}=0 ; \quad E_{1 ; 3 ; 4 ; 6}=E ; \quad E_{2 ; 5}=\alpha E\right.$ $\left.\left(\frac{1}{2}<\alpha<1\right) ; E_{0} \gg E\right]$ there are two different pairs of potential minima: "ferromagnetic" (FM) and "antiferromagnetic" $(\mathrm{AF})$, with parallel and antiparallel loop currents, respectively:

$$
\begin{gathered}
\phi_{0}^{\mathrm{FM}}=0, \\
\phi_{1 ; 3 ; 4 ; 6}^{\mathrm{FM}}= \pm \arccos (1 / 2 \alpha) \\
\phi_{0}^{\mathrm{AF}}= \pm \frac{\hbar I_{\mathrm{p}}}{e E_{0}}+\mathcal{O}\left[\left(E / E_{0}\right)^{2}\right],
\end{gathered}
$$

$$
\phi_{1 ; 3}^{\mathrm{AF}}=-\phi_{4 ; 6}^{\mathrm{AF}}= \pm \arccos (1 / 2 \alpha) \pm \frac{1-2 \alpha^{2}}{4 \alpha^{2}-1} \frac{\hbar I_{\mathrm{p}}}{e E_{0}}+\mathcal{O}\left[\left(E / E_{0}\right)^{2}\right]
$$

where $I_{\mathrm{p}}=(2 e / \hbar) E \sqrt{1-1 / 4 \alpha^{2}}$ is the persistent current in a free $3 \mathrm{JJ}$ qubit. ${ }^{2}$ Inserting these into $U_{\mathrm{J}}$, one finds that the $\mathrm{AF}$ states have the lower energy by

$$
\Delta U=2 J=\frac{\hbar^{2} I_{\mathrm{p}}^{2}}{2 e^{2} E_{0}}+\mathcal{O}\left[\left(E / E_{0}\right)^{2}\right],
$$

so that the effective mutual inductance $\hbar^{2} / 4 e^{2} E_{0}$ is just the standard Josephson inductance of the coupling junction. ${ }^{8}$ For an explanation, note that flipping the signs of $\phi_{4 ; 6}^{\mathrm{FM}}$ yields an

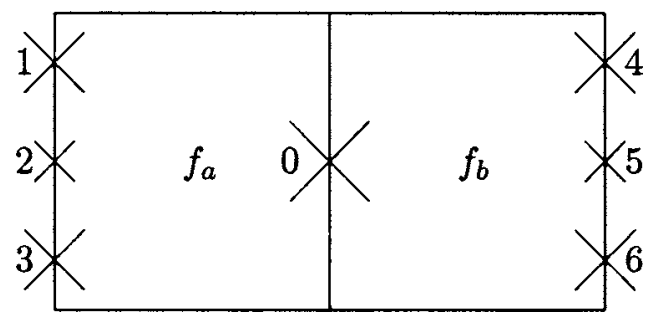

FIG. 1. Schematics of a 7JJ device: two 3JJ qubits with direct Josephson coupling and reduced flux bias $f_{a ; b}$. 


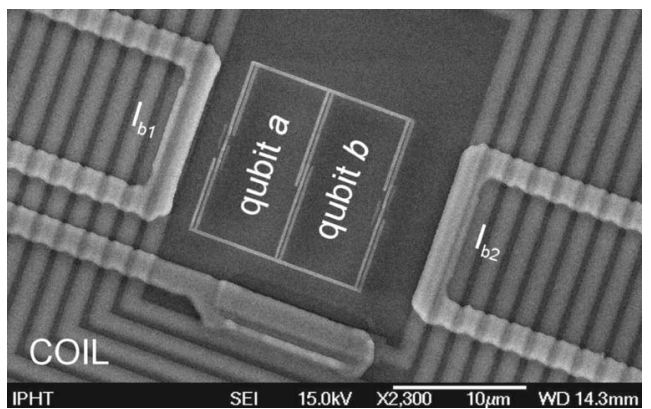

FIG. 2. Micrograph of sample 1 (see Table I). The 3JJ qubits $a$ and $b$ are coupled through a shared Josephson junction, visible in the center; they can be flux biased independently by dc lines $I_{\mathrm{b} 1}$ and $I_{\mathrm{b} 2}$. The pancake coil is part of an $L C$ circuit reading out the qubits' magnetic susceptibility.

AF configuration with $\phi_{0}=0$, and with the same energy as the FM minimum. Such a state of course is nonstationary (since charge must be conserved), and adjustment of the phases will lower $U_{\mathrm{J}}$, with the stationary AF state (2) realizing the global minimum. More intuitively: the nonzero $\phi_{0}^{\mathrm{AF}}$ reduces the effective frustration in the individual qubits, which is maximal for $f_{a ; b}=0$ [cf. above (1)]; the attendant reduction in qubit energy overcomes (by a factor of 2) the increase in Josephson energy in the coupling junction itself.

Thus the direct Josephson coupling of $3 \mathrm{JJ}$ qubits has the same sign as their inductive coupling (the latter corresponding to the natural north-south alignment of their magnetic moments), ${ }^{9}$ but is not restricted by the geometric inductances. For $E_{0} \rightarrow \infty, J$ in (3) disappears as it should, since then we have two qubits sharing a common leg without a junction. In our approximation, this is equivalent to two adjacent qubits, with only inductive coupling. ${ }^{10}$ In reality, the shared leg's kinetic inductance will make a small contribution in (3); this is negligible for our samples, but it will also contribute to a finite $J\left(E_{0} \rightarrow \infty\right)$.

We determined $J$ using an impedance measurement technique, applied previously to $3 \mathrm{JJ}$ qubits ${ }^{4,11}$ and extended to multiple qubits in Ref. 12. The qubits are placed inside a tank circuit with known self-inductance $L_{\mathrm{T}}$ and quality factor $Q_{\mathrm{T}}$, driven by a dc bias plus a small ac current at its resonance frequency $\omega_{\mathrm{T}}$ (Fig. 2). The tank's voltage-current phase angle $\theta$ is given by $\tan \theta=-\left(Q_{\mathrm{T}} / L_{\mathrm{T}}\right) \chi^{\prime}$. Here, $\chi^{\prime}$ is the qubits' contribution to the tank susceptibility, ${ }^{13}$ readily related to the curvature of their energy bands: for qubit-tank mutual inductances $M_{a \mathrm{~T}}=M_{b \mathrm{~T}} \equiv M$, one has ${ }^{14}$

$$
\tan \theta=\frac{Q_{\mathrm{T}}}{L_{\mathrm{T}}} M^{2} \frac{d^{2} E_{\mathrm{tot}}}{d\left(\Phi^{\mathrm{x}}\right)^{2}},
$$

where $\Phi^{\mathrm{x}}$ denotes a symmetric change of flux bias in both qubit loops. At temperature $T=0, E_{\text {tot }}$ is the qubits' groundstate energy; at finite $T$, the derivative simply becomes a Boltzmann average over the levels. The band curvature is large near anticrossings, so that $\tan \theta\left(f_{a}, f_{b}\right)$ contains important information about the level structure.

One obtains $J$ from such a plot as follows. The standard four-state Hamiltonian for two coupled qubits is

$$
H=-\epsilon_{a} \sigma_{a}^{z}-\Delta_{a} \sigma_{a}^{x}-\epsilon_{b} \sigma_{b}^{z}-\Delta_{b} \sigma_{b}^{x}+J \sigma_{a}^{z} \sigma_{b}^{z},
$$

where $\sigma^{x}, \sigma^{z}$ are Pauli matrices, $\Delta_{j}$ is the tunneling amplitude, and $\epsilon_{j}=I_{\mathrm{p} j} \Phi_{0} f_{j}$ is the energy bias $(j=a, b)$. For low $T$ and small $\Delta_{j}$, the location of the peaks in $|\tan \theta|$ (due to anticrossings) follows simply from the classical stability diagram, showing which flux states minimize (5) for $\Delta_{a ; b}=0$ as $\epsilon_{a ; b}$ are varied. For instance, the $|\uparrow \downarrow\rangle \leftrightarrow|\uparrow \uparrow\rangle$ transition $(|\uparrow \downarrow\rangle$ : $\sigma_{a}^{z}=-\sigma_{b}^{z}=1$, etc.) occurs at $-\epsilon_{a}+\epsilon_{b}-J=-\epsilon_{a}-\epsilon_{b}+J \Rightarrow \epsilon_{b}=J$. Therefore, the peak-to-peak distance in $\epsilon_{a}$ or $\epsilon_{b}$ equals $2 J$.

For our samples, we first fabricate niobium $(\mathrm{Nb})$ pancake coils and dc flux-bias lines on 4-in. oxidized silicon wafers, and then the qubits inside the coils by aluminium (Al) shadow evaporation on $12 \times 12 \mathrm{~mm}^{2}$ chips.

The $\mathrm{Nb}$ process starts with sputtering and dry etching of the 200-nm-thick coil windings with $1-\mu \mathrm{m}$ width, $1-\mu \mathrm{m}$ line spacing, and typically 30 turns. The patterning uses $e$-beam lithography and a $\mathrm{CF}_{4}$ reactive-ion etching process. Then, a silicon oxide isolation layer and the second 300-nm-thick Nb film are deposited for the central coil electrode and the $2-\mu \mathrm{m}$-wide dc lines; photolithography is used for all required resist masks of these layers. Finally, $400 \mathrm{~nm}$ silicon oxide is deposited for protection and isolation.

The Al process uses $e$-beam lithography to prepare the double-layer resist mask for the qubits with a 150-nm linewidth. The two Al layers are deposited in situ by $e$-beam evaporation with different angles of incidence at a rate of $1.8 \mathrm{~nm} / \mathrm{s}$. The surface of the first $\mathrm{Al}$ film is oxidized with pure oxygen at a pressure of $10^{-2}$ mbar. The qubits are completed after the final lift off.

Results are shown in Fig. 3(a). As explained below (5), one has $J \approx I_{\mathrm{p} a} \Phi_{0} \delta f_{a}=I_{\mathrm{p} b} \Phi_{0} \delta f_{b}$. One can find $I_{\mathrm{p} a ; b}$ in two different ways, which agree to within $\sim 20 \%$. First, we used $I_{\mathrm{p}} \approx I_{\mathrm{c}} \sqrt{1-1 / 4 \alpha^{2}}$ [cf. below (2)]. Here, $I_{\mathrm{c}}$ is the critical current of a junction fabricated on the same chip and with the same area of $650 \times 150 \mathrm{~nm}^{2}$ as junctions $1 / 3 / 4 / 6$, enclosed in a superconducting loop and measured by the conventional rf-SQUID technique (SQUID: superconducting quantum interference device); $\alpha \approx 0.75$ by design. The second way is to fit the shape of the peaks in $\tan \theta\left(f_{a}, f_{b}\right)$ [Fig. 3(b)], using the spectrum of (5) to evaluate (4), which yields $\Delta_{j}$ and $I_{\mathrm{p} j} \cdot{ }^{11,12,15}$ The required tank parameters were extracted from its resonance characteristic; the mutual inductances follow accurately from the tank current needed to induce a quantum of flux in the qubits; for sample $1, L_{\mathrm{T}}=136 \mathrm{nH}$, $Q_{\mathrm{T}}=664, \quad \omega_{\mathrm{T}} / 2 \pi=19.925 \mathrm{MHz}$, and $M_{a \mathrm{~T}}=M_{b \mathrm{~T}}=66.5 \mathrm{pH}$. The $|\uparrow \downarrow\rangle \leftrightarrow|\downarrow \uparrow\rangle$ anticrossing does not show up in the figure because there is no net flux change, hence no contribution to the qubit susceptibility; one can also say that the level curvature is maximal in the direction perpendicular to the symmetric one stipulated in (4).

The results of the fit are summarized in Table I for two measured two-qubit samples, with different sizes of junction 0 . Note how, say, $\Delta_{a}<\Delta_{b}$ for sample 1 makes the $a$-anticrossing sharper, resulting in a deeper color for the corresponding peak (vertical bands in Fig. 3). Since $E_{0} / E$ $=3.1$ and 1.5 , respectively, the perturbative analysis leading to (3) does not apply quantitatively (the latter would have required large coupling junctions, which proved difficult to 

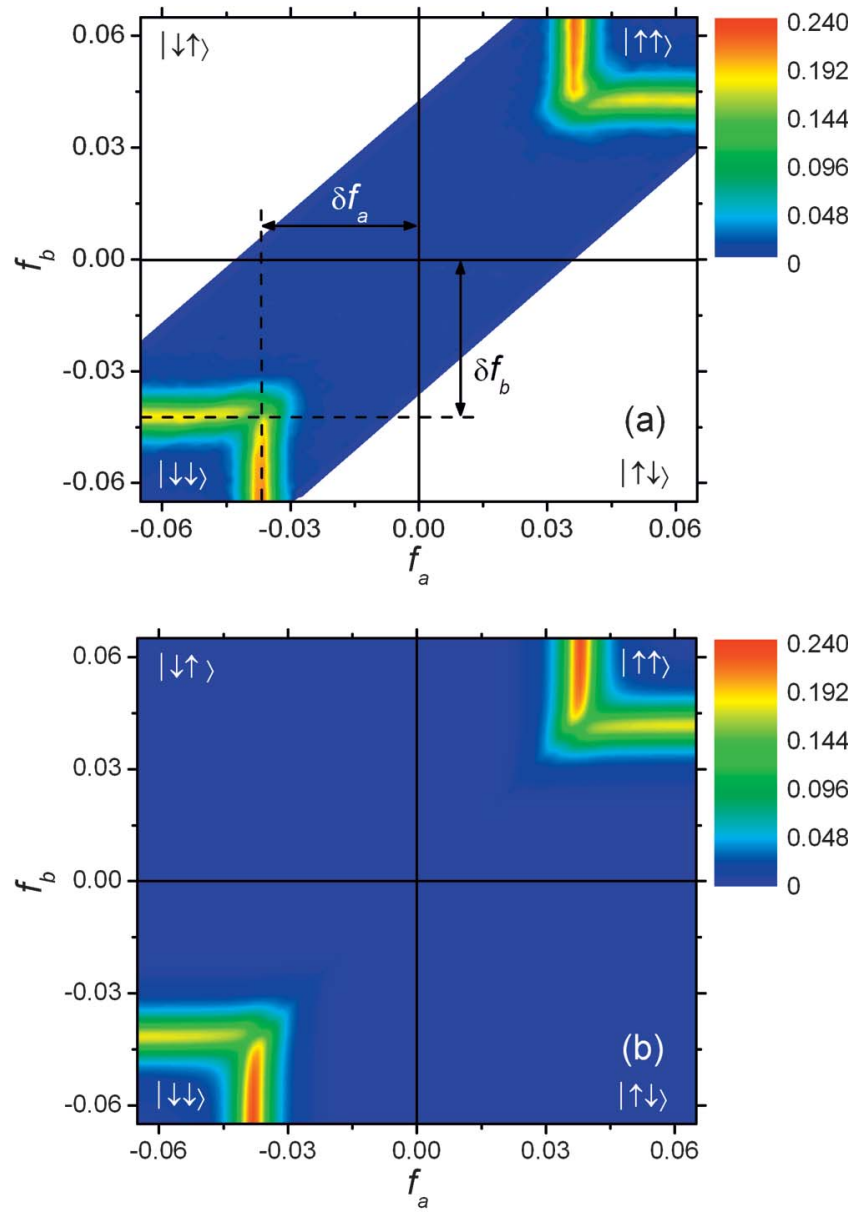

FIG. 3. (Color) Plot of $-\tan \theta\left(f_{a}, f_{b}\right)$ for two coupled 3JJ qubits (sample 1 from Table I). (a) Measurements at a nominal mixingchamber temperature of $20 \mathrm{mK}$. (b) Theoretical fit for $T=70 \mathrm{mK}$ (see the text for the different $T$ 's). The coupling strength can be estimated from the separation between the peaks.

make with sufficient homogeneity). Instead, a theoretical prediction is made for $\delta f\left(E_{0} / E, \alpha\right)$, by calculating the classical stability diagram directly from $U_{\mathrm{J}}\left(\phi_{0}, \phi_{1}, \phi_{3}, \phi_{4}, \phi_{6}\right)$. Incidentally, this has the advantage that the critical-current density drops out of the comparison (entries for $\delta f$ in the table), which therefore shows greater accuracy than we can claim for $J$ itself.

Data taken at a higher $T$ support the effective Hamiltonian (5) for our 7JJ system beyond the ground state. Namely, for, say, $f_{a} \lesssim 0.035, f_{b} \approx 0.042$, an $|\uparrow \downarrow\rangle \leftrightarrow|\uparrow \uparrow\rangle$ anticrossing persists between excited states of sample 1 . At finite $T$, it should contribute in (4), with rapidly decreasing Boltzmann weight as $f_{a}$ is reduced. This is precisely what is

TABLE I. Coupling-junction areas $S_{0}$, tunneling amplitudes $\Delta_{j}$, persistent currents $I_{\mathrm{p} j}$, peak locations $\delta f$, and coupling energies $J$ for the measured samples.

\begin{tabular}{ccccccccc}
\hline \hline $\begin{array}{c}\text { Sample } \\
\text { no. }\end{array}$ & $\begin{array}{c}S_{0} \\
\left(\mu \mathrm{m}^{2}\right)\end{array}$ & $\begin{array}{c}\Delta_{a} \\
(\mathrm{mK})\end{array}$ & $\begin{array}{c}\Delta_{b} \\
(\mathrm{mK})\end{array}$ & $\begin{array}{c}I_{\mathrm{p} a} \\
(\mathrm{nA})\end{array}$ & $\begin{array}{c}I_{\mathrm{p} b} \\
(\mathrm{nA})\end{array}$ & $\delta f_{\exp }$ & $\delta f_{\text {th }}$ & $\begin{array}{c}J \\
(\mathrm{~K})\end{array}$ \\
\hline 1 & 0.30 & 80 & 90 & 120 & 110 & $0.037-0.041$ & 0.0360 & 0.7 \\
2 & 0.15 & 30 & 30 & 150 & 120 & 0.068 & 0.0675 & 1.2 \\
\hline \hline
\end{tabular}
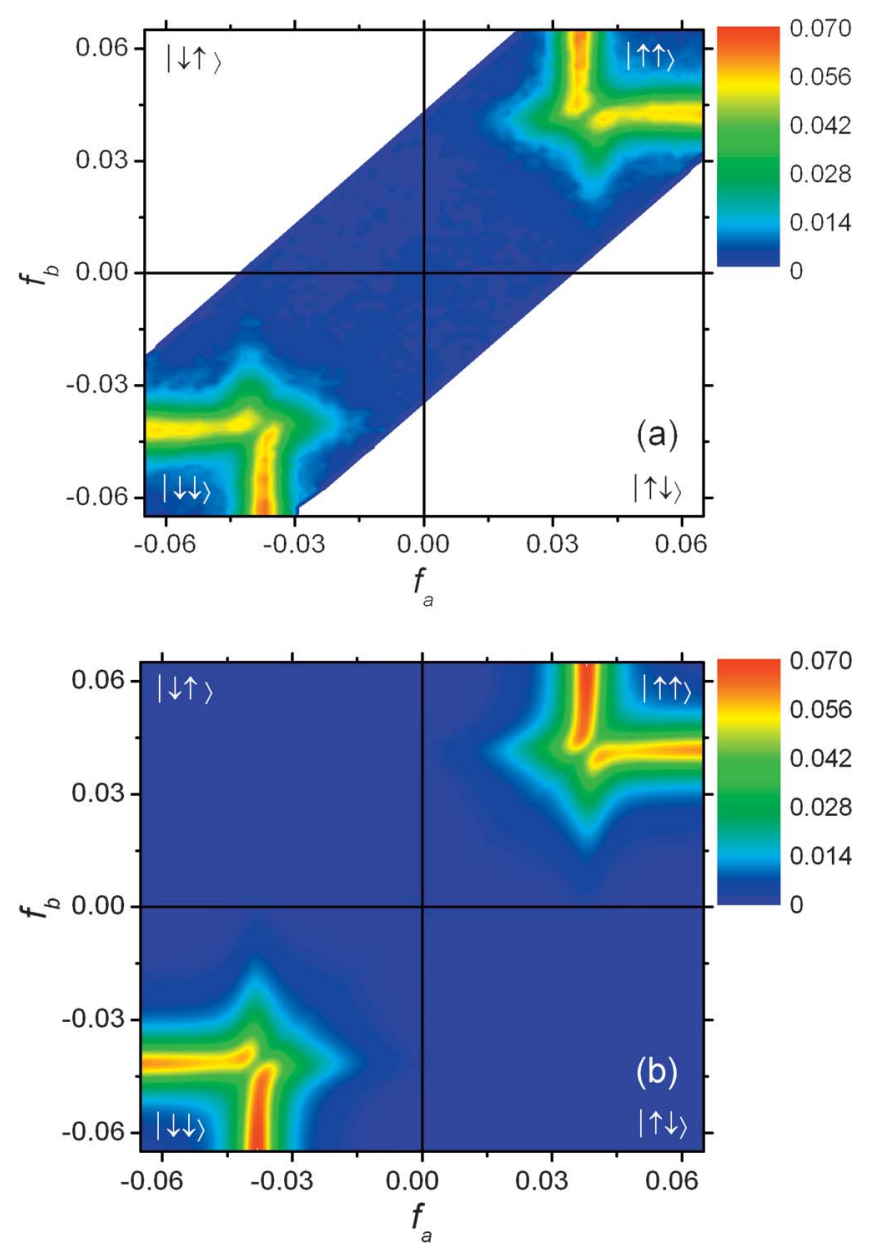

FIG. 4. (Color) As in Fig. 3, but for a mixing-chamber temperature of $200 \mathrm{mK}$ (a) and an effective $T=300 \mathrm{mK}$ (b).

seen in Fig. 4(a); the fit in Fig. 4(b) shows detailed agreement with the theory. In both Figs. 3 and 4, the discrepancy between effective and mixing-chamber temperatures is well within the range expected due to heating through external leads etc.; we observed no significant deviations from an equilibrium distribution.

The remarkable $J \sim 1 \mathrm{~K}$ significantly exceeds both the tunnel splitting and the inductive coupling (estimated to be $\sim 20 \mathrm{mK}$ ). It can be flux tuned by using a standard compound junction (dc SQUID) for the coupling. Instead, one can also apply a bias current $I_{\mathrm{b}}$ through junction 0 (Refs. 16 and 17). The corresponding generalization of Eq. (3) is

$$
J=\frac{\hbar I_{\mathrm{p}}^{2}}{2 e \sqrt{I_{\mathrm{c} 0}^{2}-I_{\mathrm{b}}^{2}}}+\mathcal{O}\left[\left(E / E_{0}\right)^{2}\right] .
$$

Thus, $J$ can only be increased, albeit significantly. Hence, this mechanism does not allow, e.g., changing the coupling sign and tunable decoupling of qubits. These are desirable for most quantum algorithms, but existing proposals for flux qubits rely on, and therefore are limited by, mutual inductances. ${ }^{18} \mathrm{~A}$ bias line will introduce some noise. For reference, we give the coupling linewidth due to lowfrequency fluctuations in $I_{\mathrm{b}}$ with spectral density $S_{\mathrm{b}}(\omega): \Delta J$ $=\hbar I_{\mathrm{p}}^{4} I_{\mathrm{b}}^{2} S_{\mathrm{b}}(0) / 4 e^{2}\left(I_{\mathrm{c} 0}^{2}-I_{\mathrm{b}}^{2}\right)^{3}$. 


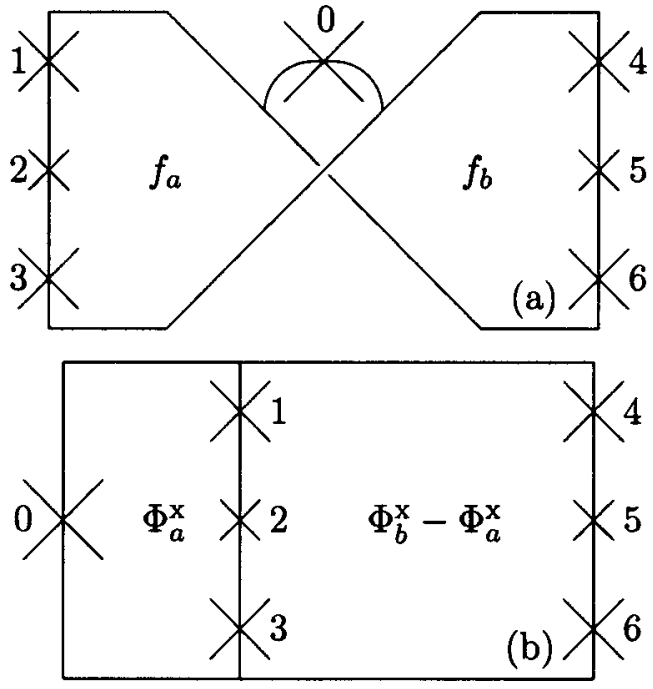

FIG. 5. Two 3JJ qubits with ferromagnetic Josephson coupling. (a) Twisted design. (b) Overlapping design.

Other variations are presented in Fig. 5. In Fig. 5(a), the relative twist between the qubit loops interchanges the role of the AF and FM configurations, so that the latter have the lowest energy. In particular, the strength of the direct FM Josephson coupling can overcome any residual AF inductive interaction. Junction 0 can presumably be fabricated between the crossing lines in the center. In Fig. 5(b), one qubit loop is
01230 and the other is 04560 ; by choosing 1:2 area ratios, both qubits can be brought close to degeneracy with a homogeneous field, for $\Phi_{a}^{\mathrm{x}}=\frac{1}{3} \Phi_{b}^{\mathrm{x}}=\frac{1}{2} \Phi_{0}$. One obtains FM coupling without a twisted layout, but with strongly asymmetric qubits. Alternatively, the right loop in Fig. 5(b) can be kept small, and used only for small bias fluxes; this is the Josephson-inductance-based counterpart of Fig. 1(b) in Ref. 19. The two discussed modifications can be combined: by current biasing the junctions 0 in Fig. 5, one obtains tunable FM coupling.

In conclusion, we have demonstrated direct antiferromagnetic Josephson coupling between two individually controllable three-junction flux qubits. The coupling strength can be on the order of a Kelvin, and agrees with theoretical predictions to the expected accuracy. We also proposed design modifications allowing tunable and/or ferromagnetic coupling. Future experimental work should also consider linear qubit arrays, to which the design of Fig. 1 is readily generalized. $^{7}$

E.I. thanks the EU for support through the RSFQubit project. S.v.d.P. thanks the ESF PiShift programme for a grant. M.G. acknowledges support by Grant No. VEGA 1/2011/05. A.Z. acknowledges support by the NSERC Discovery Grants Program. A.M.B. and A.Z. thank M.H.S. Amin, A.Yu. Smirnov, and M.F.H. Steininger for fruitful discussions.
*Electronic address: ilichev@ipht-jena.de

'Electronic address: alec@dwavesys.com

†Currently at UBC; email address: zagoskin@physics.ubc.ca

${ }^{1}$ J. R. Friedman, V. Patel, W. Chen, S. K. Tolpygo, and J. E. Lukens, Nature (London) 406, 43 (2000); C. H. van der Wal, A. C. J. ter Haar, F. K. Wilhelm, R. N. Schouten, C. J. P. M. Harmans, T. P. Orlando, S. Lloyd, and J. E. Mooij, Science 290, 773 (2000).

${ }^{2}$ J. E. Mooij, T. P. Orlando, L. Levitov, L. Tian, C. H. van der Wal, and S. Lloyd, Science 285, 1036 (1999).

${ }^{3}$ I. Chiorescu, Y. Nakamura, C. J. P. M. Harmans, and J. E. Mooij, Science 299, 1869 (2003).

${ }^{4}$ E. Il'ichev, N. Oukhanski, A. Izmalkov, Th. Wagner, M. Grajcar, H.-G. Meyer, A. Yu. Smirnov, A. Maassen van den Brink, M. H. S. Amin, and A. M. Zagoskin, Phys. Rev. Lett. 91, 097906 (2003)

${ }^{5}$ P. Bertet, I. Chiorescu, G. Burkard, K. Semba, C. J. P. M. Harmans, D. P. DiVincenzo, and J. E. Mooij, cond-mat/0412485 (unpublished).

${ }^{6}$ L. S. Levitov, T. P. Orlando, J. B. Majer, and J. E. Mooij, condmat/0108266 (unpublished).

${ }^{7}$ J. R. Butcher, Master's thesis, Delft University of Technology, 2002.

${ }^{8}$ By considering "black box" devices in the outer arms, $U_{\mathrm{J}}=U_{a}\left(2 \pi\left(\frac{1}{2}+f_{a}\right)-\phi_{0}\right)+U_{b}\left(2 \pi\left(\frac{1}{2}+f_{b}\right)+\phi_{0}\right)-E_{0} \cos \phi_{0}$, one readily verifies that the latter result also holds if the two qubits are asymmetric, biased, or of a different type. Defining the proper phase-dependent Josephson inductance at the working point, also (6) for the biased case is seen to be of this form.
${ }^{9}$ Indeed, the remarks below (3) show the derivation to be analogous to the inductive case; see A. Maassen van den Brink, Phys. Rev. B 71, 064503 (2005).

${ }^{10}$ J. B. Majer, F. G. Paauw, A. C. J. ter Haar, C. J. P. M. Harmans, and J. E. Mooij, Phys. Rev. Lett. 94, 090501 (2005).

${ }^{11}$ M. Grajcar, A. Izmalkov, E. Il'ichev, Th. Wagner, N. Oukhanski, U. Hübner, T. May, I. Zhilyaev, H. E. Hoenig, Ya. S. Greenberg, V. I. Shnyrkov, D. Born, W. Krech, H.-G. Meyer, A. Maassen van den Brink, and M. H. S. Amin, Phys. Rev. B 69, 060501(R) (2004).

${ }^{12}$ A. Izmalkov, M. Grajcar, E. Il'ichev, Th. Wagner, H.-G. Meyer, A. Yu. Smirnov, M. H. S. Amin, A. Maassen van den Brink, and A. M. Zagoskin, Phys. Rev. Lett. 93, 037003 (2004).

${ }^{13}$ Properly, $\chi^{\prime}$ is the real part, effective at $\omega_{\mathrm{T}}$.

${ }^{14}$ Ya. S. Greenberg, A. Izmalkov, M. Grajcar, E. Il'ichev, W. Krech, H.-G. Meyer, M. H. S. Amin, and A. Maassen van den Brink, Phys. Rev. B 66, 214525 (2002).

${ }^{15}$ A. Yu. Smirnov, cond-mat/0312635 (unpublished).

${ }^{16}$ For example, see J. Lantz, M. Wallquist, V. S. Shumeiko, and G. Wendin, Phys. Rev. B 70, 140507(R) (2004).

${ }^{17}$ For a nonzero leading-order $\phi_{0}=\arcsin \left(I_{\mathrm{b}} / I_{\mathrm{c} 0}\right)$, the qubits are effectively at degeneracy if $2 \pi f_{a}-\phi_{0}=2 \pi f_{b}+\phi_{0}=0$. See above (1) and note 8 .

${ }^{18}$ B. L. T. Plourde, J. Zhang, K. B. Whaley, F. K. Wilhelm, T. L. Robertson, T. Hime, S. Linzen, P. A. Reichardt, C.-E. Wu, and J. Clarke, Phys. Rev. B 70, 140501(R) (2004); A. Maassen van den Brink and A. J. Berkley, cond-mat/0501148 (unpublished).

${ }^{19}$ J. Q. You, Y. Nakamura, and F. Nori, Phys. Rev. B 71, 024532 (2005). 